(0.55\%). During 2009-2010, there are parallel trends in the detection of these two infections, but in September the reciprocal event was shown. This discrepancy may be due to the peculiarities of various microorganisms, and the clinical signs of the infectionchlamydia can cause non-gonococcal urethritis, which is the reason for examination, and mycoplasma infection is mainly symptomless. Nevertheless, a hypothesis of various patterns of infections prevalence around a year in northern countries (ie, Canada, Alaska, Scandinavia, Russia) needs further clarification. The post-holiday period (September-October) may be a crucial point in the activity of the infectious process.

\section{P1-S1.06 RECTAL HEALTH: PREVALENCE OF RECTAL STIS AND ASSOCIATIONS WITH CLINICAL SIGNS AND SELF-REPORTED SYMPTOMS}

doi:10.1136/sextrans-2011-050108.6

${ }^{1} \mathrm{P} M$ Gorbach, ${ }^{1} \mathrm{M}$ Javanbakht, ${ }^{2} \mathrm{E}$ Fuchs, ${ }^{1} \mathrm{R} \mathrm{E}$ Weiss, ${ }^{3} \mathrm{M}$ Hezerah, ${ }^{3} \mathrm{~S}$ Brown, ${ }^{1} \mathrm{~A}$ Voskanian, ${ }^{1} \mathrm{P}$ Anton, ${ }^{4} \mathrm{R}$ Cranston. ${ }^{1}$ University of California, Los Angeles, Los Angeles, USA; ${ }^{2}$ Johns Hopkins School of Medicine, USA; ${ }^{3}$ AIDS Research Alliance, USA; ${ }^{4}$ University of Pittsburgh School of Medicine, USA

Background Predictors of rectal health are needed for public health programs and planning rectal microbicide clinical trials.

Methods From October 2006 to December 2008, 896 men and women in Los Angeles and Baltimore in a rectal health and behaviours study (UCLA IPCP U19 0606414) completed interviews and rectal sexually transmitted infections (STIs) testing including Gonorrhoea (GC), Chlamydia (CT) and high-risk human papillomavirus (HR-HPV). Rectal signs detected by High Resolution Anoscopy (HRA) and self-reports of symptoms in the past month were analysed for associations with STIs (GC $(n=7)$, CT $(n=15)$ and HR-HPV $(n=178)$ ), demographics and receptive anal intercourse (RAI) in the past month. Those with active haemorrhoids were excluded $(n=77)$ from analyses of STI predictors.

Results SIGNS-Men reporting RAI $(n=234)$ had a higher prevalence of swelling seen by HRA than men not reporting RAI $(6.4 \%$ vs $1.9 \%$; $p$ value 0.02 ). Prevalence of erythema or haemorrhoids was lower among men reporting RAI ( $2.2 \%$ vs $6.8 \%$; $p$ value $=0.02$ and $18.3 \%$ vs $30.8 \%$; $p$ value $<0.01$ respectively). There were no differences in signs between women by RAI. More erythema and discharge were HRA detected among HIV-positive men; more HIV positive men and women than HIV negative had ulceration. SYMPTOMS-Significantly more RAI+ men reported symptoms (swelling, itching, burning and irritation) but there were no differences in reported symptoms among women by RAI. HIV positive men $(n=218)$ and women $(n=186)$ reported more symptoms (swelling, itching, discharge and burning) than HIV negative men and women; HIV positive men reported more pain and irritation than HIV negative men $(n=227)$. STIs - No HRA detected signs were associated with rectal GC or CT. Bleeding (OR 5.06, 95\% CI 1.09 to 23.45) and leukoplakia (OR 5.06 95\% CI 1.22 to 25.66) were significantly associated with detection of HR-HPV. No symptoms were associated with GC or CT; discharge in the past month was associated with HR-HPV (OR 2.16, 95\% CI 1.07 to 4.35).

Conclusions Lack of association between signs or symptoms and prevalent rectal STI suggests syndromic approaches may be problematic requiring laboratory testing for detection of rectal STIs. Prevalence of signs varied little by RAI practice but there was a higher prevalence of self-reported symptoms among men reporting RAI. These findings can serve as a reference for expected rates to be found in clinical trials testing rectal microbicides and interpretation of adverse events.

\section{P1-S1.07 MULTIPLE BACTERIAL SEXUALLY TRANSMITTED INFECTIONS IN ONTARIO, CANADA}

doi:10.1136/sextrans-2011-050108.7

${ }^{1} \mathrm{C}$ Lee, ${ }^{1} \mathrm{M}$ Whelan, ${ }^{2} \mathrm{C}$ Achonu, ${ }^{1} \mathrm{~W}$ Bhanich-Supapol, ${ }^{2} \mathrm{~J}$ Christian. ${ }^{1}$ Ontario Ministry of Health and Long-Term Care, Toronto, Canada; ${ }^{2}$ Ontario Agency for Health Protection and Promotion, Canada

Background In 2009, there were 33000 reported cases of chlamydia, gonorrhoea and infectious syphilis in Ontario. These reportable bacterial sexually transmitted infections (STIs) represented approximately $48 \%$ of all reportable disease cases reported in Ontario that year. A significant amount of resources is expended on public health case and contact management of bacterial STIs. The objective of our study was to use routine surveillance data from Ontario to quantify the incidence of persons with reported multiple bacterial STIs in Ontario from 2006 to 2009 in order to inform future public health interventions.

Methods All bacterial STI records from 2006 to 2009 were extracted from Ontario's integrated Public Health Information System (iPHIS). Multiple STIs were defined as repeat bacterial STIs or infection with a different bacterial STI in the same individual. Repeat STIs were defined as diagnoses of chlamydia or gonorrhoea more than 28 days after the previous infection. The data were analysed using PASW 18 for Windows (SPSS Inc.)

Results There were almost 100000 unique clients with at least one bacterial STI from 2006 to 2009 representing 113097 STI cases. Approximately $24 \%$ of STI cases reported from 2006 to 2009 occurred in individuals with multiple STIs during the same timeframe. However, clients with multiple STIs accounted for only $12 \%$ of the total number of clients reporting at least one STI in this 4year time period and clients with three or more STIs only accounted for $2.4 \%$ of all clients. The majority of clients $(\sim 60 \%)$ with multiple STIs were infected within one year of their first STI. On average clients with multiple STIs had more sexual contacts recorded for their first STI than those clients who only had one STI (1.44 contacts vs 1.33 contacts, $\mathrm{p}<0.001)$. There was no difference in the number of clients lost to follow-up when comparing clients with only one STI to those with multiple STIs ( $p>0.9)$.

Conclusions Public health resources may be well spent in case and contact management because almost $90 \%$ of individuals only have one STI reported. However, individuals who have multiple STIs account for a substantial proportion of reported bacterial STI cases in Ontario. Further work to identify additional characteristics of individuals at risk for multiple STIs would aid in informing future public health interventions aimed at these high-risk individuals.

\section{P1-S1.08 PREVALENCE OF NEISSERIA GONORRHOEAE AND CHLAMYDIA TRACHOMATIS INFECTION IN MEN ATTENDING STD CLINICS IN BRAZIL}

doi:10.1136/sextrans-2011-050108.8

${ }^{1} \mathrm{M}$ Barbosa, ${ }^{2} \mathrm{~V}$ M Pinto, ${ }^{2} \mathrm{~F}$ Moherdaui, ${ }^{2} \mathrm{D}$ Ribeiro, ${ }^{3} \mathrm{~A}$ Espinosa. ${ }^{1}$ Federal District Government, Brasilia, Brazil; ${ }^{2}$ Ministry of Health, Brazil; ${ }^{3}$ UFES, Brazil

Introduction The study aimed to assess the prevalence of Neisseria gonorrhoeae and Chlamydia trachomatis infections and identify demographic, behavioural and clinical factors correlated with such infections in men attending six sexually transmitted disease clinics in Brazil.

Methods Multicentric, cross-sectional study performed among men attending STD clinics in Brazil. The study included STD clinics in six cities distributed throughout the five geographic regions of Brazil in 2005. Patients provided $20 \mathrm{ml}$ of first catch urine for testing for $N$ gonorrhoeae and $C$ trachomatis by DNA-PCR. 